\title{
The Design of Linear Algebra and Geometry
}

\author{
David Hestenes
}

\begin{abstract}
Conventional formulations of linear algebra do not do justice to the fundamental concepts of meet, join, and duality in projective geometry. This defect is corrected by introducing Clifford algebra into the foundations of linear algebra. There is a natural extension of linear transformations on a vector space to the associated Clifford algebra with a simple projective interpretation. This opens up new possibilities for coordinate-free computations in linear algebra. For example, the Jordan form for a linear transformation is shown to be equivalent to a canonical factorization of the unit pseudoscalar. This approach also reveals deep relations between the structure of the linear geometries, from projective to metrical, and the structure of Clifford algebras. This is apparent in a new relation between additive and multiplicative forms for intervals in the cross-ratio. Also, various factorizations of Clifford algebras into Clifford algebras of lower dimension are shown to have projective interpretations.

As an important application with many uses in physics as well as in mathematics, the various representations of the conformal group in Clifford algebra are worked out in great detail. A new primitive generator of the conformal group is identified.
\end{abstract}

[Note: Some corrections have been added in brackets.]

\section{Introduction}

It is sometimes said that projective geometry is just linear algebra. Nevertheless, the two subjects persist today as separate branches of mathematics. Although they grew up together in the nineteenth century, projective geometry was left behind as linear algebra flourished through diverse applications in the twentieth century. This is the second of two articles aimed at bringing them back together in a mutually beneficial reconciliation. These articles propose specific designs for a mathematical system to achieve that end.

Conventional linear algebra is based on the concepts of vector space and linear transformation. Projective geometry can be formulated within this framework, but only by introducing coordinate systems without intrinsic geometric significance. To achieve a coordinatefree formulation of projective geometry, a richer algebraic system is needed. In the first article [1] geometric algebra was proposed as the ideal system for that purpose. It was shown to have exactly the right dimension and algebraic structure to describe all the meet and join operations essential to projective geometry. This article extends the mathematical designs of [1] along two lines: (1) The standard concept of a linear transformation is extended to provide a direct, coordinate-free formulation of projective transformations, and this extension is shown to be valuable even in standard linear algebra. As an example, the Jordan canonical form is shown to be equivalent to a particular factoring of the unit pseudoscalar. (2) The concepts of 'projective split' and 'conformal split' are introduced 
and shown to reveal a deep connection between projective structures and the structure of Clifford algebras. They are used to simplify and coordinate relations between projective, conformal, affine, and metric geometries. A new relation between additive and multiplicative forms for intervals in the cross-ratio is derived. The conformal groups for metric spaces of arbitrary dimension and signature are analyzed in great detail, establishing connections between several alternative approaches in the literature.

Reference [1] is taken as prerequisite to this article, so the notations and nomenclature introduced there can be taken for granted. An overview of that article and the present one has been published in [2].

\section{Outermorphism}

This section reviews the concept of outermorphism developed in [3], with emphasis on its significance for projective geometry. The treatment in [3] is more general and extensive than the present one. Unfortunately, that may have obscured the essential simplicity and utility of the outermorphism idea, so the aim here is to clarify the concept. To that end, some of the results in [3] will be introduced without proof, while others are omitted.

The outermorphism is actually inherent in linear algebra. By making it explicit and giving it a notation we can exploit it more easily. The conventional abstract formulation of linear algebra follows the format of matrix algebra. This does not do justice to Grassmann's outer product or to duality, two fundamental concepts of projective geometry. The main point of this section is that the outermorphism concept corrects this defect perfectly. Since the outermorphism is inherent in linear algebra, it has been repeatedly rediscovered in various guises. For example, as explained in [3], the so-called 'push-forward' and 'pullback' mappings of differential forms are outermorphisms. While preparing this article, it was discovered that Whitehead [4] had independently defined the outermorphism of a blade and was probably the first to do so. He evidently sensed the importance of the idea, but he did not develop it far, and no one seems to have followed his lead.

As in [1], $\mathcal{V}_{n}$ is an $n$-dimensional vector space which generates a geometric algebra $\mathcal{G}_{n}=\mathcal{G}\left(\mathcal{V}_{n}\right)$. Every linear transformation $f$ on $\mathcal{V}_{n}$ has a unique extension to a linear transformation $\underline{f}$ on $\mathcal{G}_{n}$ defined by the following four properties:

$$
\begin{gathered}
\underline{f}(A \wedge B)=(\underline{f} A) \wedge(\underline{f} B), \\
\underline{f} a=f a, \\
\underline{f} \alpha=\alpha, \\
\underline{f}(\alpha A+\beta B)=\alpha \underline{f} A+\beta \underline{f} B,
\end{gathered}
$$

where $A, B$ are blades in $\mathcal{G}_{n}, a$ is a vector and $\alpha, \beta$ are scalars. Thus, $f$ is linear on $\mathcal{G}_{n}$ and distributive with respect to the outer product. It is called the outermorphism of $f$ because, according to (2.1), it preserves the outer product.

It follows from (2.1) and (2.2) that $\underline{f}$ is step-preserving, that is,

$$
\underline{f}\left(\langle M\rangle_{k}\right)=\langle\underline{f} M\rangle_{k}
$$


for any multivector $M$ in $\mathcal{G}_{n}$, Moreover, for any factoring $A=a_{1} \wedge a_{2} \wedge \cdots \wedge a_{r}$ of an $r$-blade $A$ into vectors

$$
\underline{f} A=\left(f a_{1}\right) \wedge\left(f a_{2}\right) \wedge \cdots \wedge\left(f a_{r}\right) .
$$

That is to say, $f A$ does not depend on how $A$ might be factored into vectors. It will be seen that this property is a great help in finding and describing the invariants of a linear transformation.

For the sake of simplicity, we limit our attention here to transformations from $\mathcal{V}_{n}$ to $\mathcal{V}_{n}$, so $\underline{f}$ maps $\mathcal{G}_{n}$ into $\mathcal{G}_{n}$. In this case, $(2.5)$ implies that $\underline{f}$ can alter the pseudoscalar $I$ only by a scalar multiple. Indeed,

$$
\underline{f} I=(\operatorname{det} f) I
$$

defines the determinant of $f$, which we write as $\operatorname{det} f$ or $\operatorname{det} f$. Note that the outermorphism concept makes it possible to define the determinant of a linear transformation without introducing a basis or matrices. As shown in [3], the matrix form of the determinant is easily introduced when needed, but the determinant can often be evaluated without introducing matrices.

Let $h=g f$ be the product of linear transformations $f$ and $g$ on $\mathcal{V}_{n}$. From (2.6) it is easily proved that

$$
\underline{h}=\underline{g} \underline{f} .
$$

In other words, the outermorphism of a product is equal to the product of outermorphisms. Some important facts about linear transformations follow immediately from (2.8). For example,

$$
\underline{g} \underline{f} I=(\operatorname{det} f) \underline{g} I=(\operatorname{det} f)(\operatorname{det} g) I .
$$

Therefore,

$$
\operatorname{det}(f g)=(\operatorname{det} f)(\operatorname{det} g) .
$$

It follows by iteration that, for positive integer $k$, $\operatorname{det}\left(\underline{f}^{k}\right)=(\operatorname{det} \underline{f})^{k}$, and if $f^{-1}$ exists, then $\left(\operatorname{det} f^{-1}\right)(\operatorname{det} f)=1$.

Every linear transformation $f$ has an adjoint transformation $\bar{f}$, and the adjoint can be extended to an outermorphism which we denote by $\bar{f}$ also. The adjoint outermorphism $\bar{f}$ can be defined directly by

$$
\langle M \bar{f} N\rangle=\langle(\bar{f} N) M\rangle=\langle N \underline{f} M\rangle
$$

assumed to hold for all $M, N$ in $\mathcal{G}_{n}$. The multivector derivative defined in [3] can be used to get

$$
\bar{f} N=\nabla_{M}\langle N \underline{f} M\rangle .
$$

Differentiation is helpful and much used in [3], especially to generate invariant relations, but it is not essential and will be avoided in the following. It follows from (2.7) and (2.10) that

$$
\operatorname{det} \underline{f}=I^{-1} \underline{f} I=\left\langle I^{-1} \underline{f} I\right\rangle=I^{-1} \bar{f} I=\operatorname{det} \bar{f} .
$$

Unlike the outer product, the inner product is not generally preserved by an outermorphism. However, it is proved in [3] that the inner product obeys the fundamental transformation law

$$
A \cdot(\underline{f} B)=\underline{f}[(\bar{f} A) \cdot B] \quad \text { or } \quad(\underline{f} B) \cdot A=\underline{f}[B \cdot(\bar{f} A)],
$$


for $($ step $A) \leq($ step $B)$. For clarity, the square brackets are used as parentheses here. Note that both $\underline{f}$ and $\bar{f}$ are needed to describe how the inner product transforms. Also note that, by symmetry, $\underline{f}$ and $\bar{f}$ can be interchanged in (2.13).

The law (2.13) admits the important special case

$$
A(\bar{f} I)=\bar{f}[(\underline{f} A) I] \quad \text { or } \quad(\bar{f} I) A=\bar{f}[I(\underline{f} A)] .
$$

For $\operatorname{det} f \neq 0$, this gives us immediately an explicit expression for the inverse outermorphism:

$$
\underline{f}^{-1} A=\frac{\bar{f}(A I) I^{-1}}{\operatorname{det} f}=\frac{I^{-1} \bar{f}(I A)}{\operatorname{det} f} .
$$

Applied to $a$ in $\mathcal{V}_{n}$, of course, this gives us the inverse transformation

$$
f^{-1}(a)=\frac{I^{-1} \bar{f}(I a)}{\operatorname{det} f}
$$

This reveals that the adjoint and double duality are essential relations underlying the general matrix formula for $f^{-1}$, which can, in fact, be obtained easily from (2.16).

The inverse of the outermorphism $f$ given by (2.15) is equal to the outermorphism of the inverse $f^{-1}$. To prove that, we need only establish the distributive property

$$
\underline{f}^{-1}(A \wedge B)=\left(\underline{f}^{-1} A\right) \wedge\left(\underline{f}^{-1} B\right) .
$$

For the proof it will be convenient to use the notation $A=\widetilde{A} I^{-1}$ and put (2.14) in the form

$$
\underline{f}[(\bar{f} \widetilde{A}) I]=A \operatorname{det} f .
$$

The proof uses the duality of inner and outer productsalong with (2.13) as follows. Assuming $($ step $A)+(\operatorname{step} B) \leq n$,

$$
\begin{aligned}
\left(\underline{f}^{-1} A\right) \wedge\left(\underline{f}^{-1} B\right) & =\frac{[(\bar{f} \widetilde{A}) I] \wedge[(\bar{f} \widetilde{B}) I]}{(\operatorname{det} f)^{2}}=\frac{[(\bar{f} \widetilde{A}) I] \cdot(\bar{f} \widetilde{B})}{(\operatorname{det} f)^{2}} I \\
& =\frac{\bar{f}[(\underline{f}[(\bar{f} \widetilde{A}) I]) \cdot \widetilde{B}]}{(\operatorname{det} f)^{2}} I=\frac{\bar{f}[A \cdot \widetilde{B}] I}{\operatorname{det} f}=\frac{\bar{f}\left[(A \wedge B) I^{-1}\right]}{\operatorname{det} f} .
\end{aligned}
$$

Thus (2.17) is established. Now let us turn to the matter of geometrical interpretation.

Outermorphisms can be supplied with a geometrical interpretation by adopting the projective interpretations for blades introduced in [1]. Recall that vectors representing rays in $\mathcal{V}_{n}$ can be identified with points in the projective space $\mathcal{P}_{n-1}$. Projective transformations (or projectivities) are of two types, collineations and correlations. The collineations of $\mathcal{P}_{n-1}$ are simply linear transformations of $\mathcal{V}_{n}$ with nonvanishing determinants. A correlation is simply the composite of a collineation with a duality transformation (i.e., multiplication by a pseudoscalar). Having discussed duality in [1], we can limit our attention here to collineations without loss of generality.

The most basic fact about collineations is that they map points into points, lines into lines, planes into planes, etc. This follows from the distributive property of outermorphisms, 
since points, lines, and planes can be represented by blades of step $1,2,3$, respectively. Indeed, a collineation $f$ induces an outermorphism of the equation $x \wedge A=0$ into

$$
x^{\prime} \wedge A^{\prime}=(\underline{f} x) \wedge(\underline{f} A)=0 .
$$

This is equivalent to the original equation for point, line, or plane provided $f A \neq 0$ when $A \neq 0$, and that is assured by the fact that every nonzero blade is a factor of the pseudoscalar $I$. For if blade $A \neq 0$, there exists another (not unique) blade $B$ such that $A \wedge B=I$. Since

$$
(\underline{f} A) \wedge(\underline{f} B)=(\operatorname{det} f) I \neq 0,
$$

the factor $f A$ cannot vanish.

Thus, we see that the outermorphism $f$ of a collineation $f$ directly describes the induced transformations of lines and planes. We can interpret $\underline{f} A$ as the transformation of a specific line or plane according as blade $A$ has step 2 or 3 .

To establish the invariance of projective relations under arbitrary projectivities, we need to prove that the fundamental meet and join relations are preserved under collineations. Since we can identify the join with the outer product, the invariance of the join relation is assured by the distributive property (2.1). To find the transformation law for the meet, recall that for $(\operatorname{step} A)+(\operatorname{step} B) \geq n$, the meet can be expressed in terms of the inner product by Equation (3.6) of [1]:

$$
A \vee B=\widetilde{A} \cdot B
$$

Proceeding as in the proof of $(2.17)$, we have

$$
\begin{aligned}
(\underline{f} A) \vee(\underline{f} B) & =\left[(\underline{f} A) I^{-1}\right] \cdot(\underline{f} B)=\underline{f}\left[\left(\bar{f}\left[(\underline{f} A) I^{-1}\right]\right) \cdot B\right. \\
& =\underline{f}[\widetilde{A} \cdot B](\operatorname{det} f) .
\end{aligned}
$$

Thus, the meet obeys the transformation law

$$
(\underline{f} A) \vee(\underline{f} B)=(\operatorname{det} f) \underline{f}(A \vee B) .
$$

The factor $(\operatorname{det} f)$ here is not significant in nonmetrical applications of projective geometry, since the definition of the meet is arbitrary within a scale factor. However, it can be removed by a natural change in the definition of duality, defining duality on the left with respect to the transformed pseudoscalar $I^{\prime}=\underline{f} I=(\operatorname{det} f) I$. Then $(2.15)$ implies that the transformation $A^{\prime}=\underline{f} A$ entails the induced transformation $\widetilde{A}^{\prime}=\bar{f} \widetilde{A}$ on the dual $\widetilde{A}^{\prime}=A^{\prime}\left(I^{\prime}\right)^{-1}$, and $(2.21)$ can be put in the alternative form

$$
\underline{f}(\widetilde{A} \cdot B)=\widetilde{A}^{\prime} \cdot B^{\prime} .
$$

This transformation law is mathematically equivalent to (2.13), but its geometric meaning is much more transparent. It tells us that the 'incidence properties' in projective geometry are invariant under collineations or, equivalently, that the 'subspace intersection property' is preserved by nonsingular linear transformations. For this reason, it should be counted as one of the fundamental results of linear algebra, though it does not appear in standard textbooks on the subject. 


\section{Invariant Blades}

The outermorphism is a natural tool for characterizing the invariant subspaces of an arbitrary linear transformation. As an illustration, this section shows how to use that tool to achieve a characterization equivalent to the Jordan canonical form.

The outermorphism gives us a natural generalization of the eigenvector concept. A blade $A$ is said to be an eigenblade of $f$ with (scalar) eigenvalue $\lambda$ if

$$
\underline{f} A=\lambda A \text {. }
$$

'Projectively speaking', this says that $A$ is a fixed point, line, or plane of a collineation $f$ according as the step of $A$ is 1,2 , or 3 . Let us call $A$ a symmetric eigenblade if

$$
\underline{f} A=\bar{f} A=\lambda A \text {. }
$$

This generalizes to arbitrary linear transformations an important feature of the eigenvectors of a symmetric transformation. Of course, the pseudoscalar $I$ is a symmetric eigenblade of every linear transformation, with

$$
\underline{f} I=\bar{f} I=\mu I
$$

where $\mu=\operatorname{det} f$. Henceforth, it will be convenient to assume $\mu \neq 0$.

If $A$ is an eigenblade of $f$, then its dual $\widetilde{A}=A I^{-1}$ is an eigenblade of $\bar{f}$. This follows trivially from (2.14); specifically, with (3.1) and (3.3), we have

$$
\bar{f} \widetilde{A}=\frac{\mu}{\lambda} \widetilde{A} .
$$

Since $I=A^{-1} \widetilde{A}=A^{-1} \wedge \widetilde{A}$,

$$
\bar{f} I=\mu I=\left(\bar{f} A^{-1}\right) \wedge(\bar{f} \widetilde{A})=\frac{\mu}{\lambda}\left(\bar{f} A^{-1}\right) \wedge \widetilde{A} .
$$

We cannot in general remove the wedge from the right side of this expression, but we can conclude that there exists a blade $B$ with the same step as $A$ such that

$$
\bar{f} A=\lambda A+B .
$$

where $B \wedge \widetilde{A}=(B \cdot A) I^{-1}=0$. Dually, we have

$$
f \widetilde{A}=\frac{\mu}{\lambda} \widetilde{A}+\widetilde{C} .
$$

where $A \wedge \widetilde{C}=(A \cdot C) I^{-1}=0$. Clearly, $A$ is a symmetric eigenblade of $f$ if and only if $\widetilde{A}$ is also symmetric; moreover

$$
\underline{f} A=\bar{f} \widetilde{A}=\frac{\mu}{\lambda} \widetilde{A} .
$$

A symmetric eigenblade will be called a proper blade of $f$ if it has no factors which are symmetric eigenblades of lower step. By iterating the argument yielding (3.6), we can decompose the pseudoscalar $I$ into a geometric product of proper blades $I_{k}$; thus,

$$
I=I_{1} I_{2} \cdots I_{m},
$$


where, for $k=1,2, \ldots, m \leq n$,

$$
\underline{f} I_{k}=\bar{f} I_{k}=\mu_{k} I_{k}
$$

and

$$
\operatorname{det} \underline{f}=\mu=\mu_{1} \mu_{2} \cdots \mu_{m} .
$$

The $I_{k}$ are unique (within a scale factor) if the $\mu_{k}$ are distinct. Their supports are precisely the invariant subspaces of the linear transformation $f$. If all the $I_{k}$ have step 1 , then $m=n$ and (3.7b) is a complete spectral decomposition of $f$. However, for any $I_{k}$ with (step $\left.I_{k}\right)>1$, we must take the decomposition further to characterize $f$ completely. That we do next.

[Warning: The following analysis does not suffice to establish the Jordan form for an arbitrary linear transformations, though the method of analysis may be of general interest in linear algbera.]

Suppose that $f$ has a proper blade $A_{m}$ of step $m>1$, and let complex scalars be allowed just to avoid discussing points of peripheral interest. Then the following result is equivalent to the Jordan decomposition of $f$ : there exists a unique scalar $\lambda$ and a nested sequence of blades $A_{k}$ such that

$$
\underline{f} A_{k}=\lambda^{k} A_{k}
$$

for $k=1,2, \ldots, m$. The term 'nested' means that $A_{k-1}$ is a factor of $A_{k}$. Let $\widetilde{A}_{k}=A_{k} A_{m}^{-1}$ be the dual with respect to $A_{m}$; then (3.4) implies that

$$
\bar{f} \widetilde{A}_{k}=\lambda^{m-k} \widetilde{A}_{k} .
$$

The $A_{k}$ are unique and they completely characterize $f$ on the support of $A_{m} k$. In fact, the $A_{k}$ can be generated from the unique eigenvector $a_{1}=A_{1}$, by

$$
A_{k}=a_{1} \wedge\left(\bar{f} A_{k-1}\right)
$$

which iterates to

$$
A_{k}=a_{1} \wedge\left(\bar{f} a_{1}\right) \wedge\left(\bar{f}^{2} a_{1}\right) \wedge \cdots \wedge\left(\bar{f}^{k-1} a_{1}\right) .
$$

We can prove all this by showing that it is equivalent to the Jordan canonical form.

The blades $A_{k}$ determine $m$ vectors $a_{k}$ defined by

$$
a_{k}=A_{k-1}^{-1} A_{k}=A_{k-1}^{-1} \cdot A_{k}
$$

with the convention $A_{0}=1$. Conversely, the $A_{k}$ are determined by the $a_{k}$ according to

$$
A_{k}=A_{k-1} a_{k}=a_{1} a_{2} \cdots a_{k} .
$$

Note that this implies the orthogonality relation

$$
a_{i} \cdot a_{j}=0 \text { for } i \neq j \text {. }
$$

To ascertain the action of $f$ on the $a_{k}$, we apply (3.8) to (3.13) to obtain

$$
\lambda A_{k}=A_{k-1} \wedge\left(\underline{f} a_{k}\right) .
$$


This can be solved for $\underline{f} a_{k}=f a_{k}$ as follows:

$$
\begin{aligned}
\lambda A_{k-1}^{-1} A_{k} & =\lambda a_{k}=A_{k-1}^{-1} \cdot\left(A_{k-1} \wedge\left(\underline{f} a_{k}\right)\right) \\
& =\left(A_{k-1}^{-1} \cdot A_{k-1}\right) \underline{f} a_{k}+(-1)^{k-1}\left(A_{k-1}^{-1} \cdot\left(\underline{f} a_{k}\right)\right) \cdot A_{k-1} \\
& =\underline{f} a_{k}-a_{k-1} .
\end{aligned}
$$

The last step follows from the 'Laplace expansion'

$$
\begin{aligned}
\left(a_{k}^{-1} \wedge \cdots \wedge a_{2}^{-1} \wedge a_{1}^{-1}\right) \cdot\left(\underline{f} a_{k}\right) & =(-l)^{k-2} a_{k-2}^{-1} \cdots a_{2}^{-1} a_{1}^{-1}\left(a_{k-1}^{-1} \cdot \underline{f} a_{k}\right) \\
& =(-l)^{k-2} a_{k-2}^{-1} .
\end{aligned}
$$

Only one term in the expansion survives because

$$
a_{j}^{-1} \cdot\left(\underline{f} a_{k}\right)=a_{k}\left(\bar{f} a_{j}^{-1}\right)=0 \quad \text { for } \quad j<k-1,
$$

as follows from the vanishing of terms in the formula

$$
A_{j+1} \cdot a_{k}^{-1}=\left[A_{j} \wedge\left(\bar{f} a_{j}\right)\right] \cdot a_{k}^{-1}=A_{j}\left(a_{k}^{-1} \cdot \bar{f} a_{j}\right)-\left(A_{j} \cdot a_{k}^{-1}\right) \wedge A\left(\bar{f} a_{j}\right) .
$$

For $j=k-1$, this formula implies the relation

$$
a_{k}^{-1} \cdot\left(\bar{f} a_{k-1}\right)=a_{k-1} \cdot\left(\underline{f} a_{k}^{-1}\right)=1
$$

which was also used above.

Thus we have proved that the nested eigenblade formula (3.8) implies that

$$
\underline{f} a_{k}=\lambda a_{k}+a_{k-1}
$$

for $1<k \leq m$. Of course,

$$
\underline{f} a_{1}=\lambda a_{1}
$$

since $a_{1}=A_{1}$. Note that no assumptions about signature were used in the proof, although it was necessary that $A_{m}^{2} \neq 0$. Conversely, (3.8) can be derived from (3.14b,c) simply by inserting them into

$$
\underline{f} A_{k}=\left(\underline{f} a_{1}\right) \wedge\left(\underline{f} a_{2}\right) \wedge \cdots \wedge\left(\underline{f} a_{k}\right) .
$$

Equations (3.14a,b,c) will be recognized as the Jordan canonical form for a linear transformation on a cyclic subspace. We have proved that on the support of $A_{m}$ the Jordan form is equivalent to the factoring of $A_{m}$ into a nested sequence of eigenblades.

If only real scalars are allowed, the above decomposition of a cyclic subspace applies if the characteristic polynomial has a root $\lambda$ of multiplicity $m$, in which case there is exactly one eigenvector. However, when the characteristic polynomial of the subspace does not have a real root, it has an irreducible factor of some multiplicity $m$. In this case, the subspace necessarily has even dimension $2 m$. There are no eigenvectors in the subspace, but there is, nevertheless, a scalar $\gamma$ and a nested sequence of blades $A_{2 k}$ with even step $2 k$ such that

$$
\underline{f} A_{2 k}=\gamma^{k} A_{2 k}
$$

for $k=1,2, \ldots, m$. This assertion can be proved along lines similar to the previous case, but we will not go into that here. It should be noted, though, that the determination of the eigenblades is not sufficient, in this case, to characterize the linear transformation completely. 


\section{Projective Splits}

We have seen how the entire geometric algebra $\mathcal{G}_{n+1}$ is needed to describe the join, meet, and duality operations on the projective $n$-space $\mathcal{P}_{n}$ as well as their projective outermorphisms. This section shows that the multiplicative structure of geometric algebra reflects deep properties of projective geometry and facilitates connections with affine and metric geometry.

The reduction of projective geometry to affine geometry can be expressed as a relation between a vector space $\mathcal{V}_{n+1}$ and a vector space $\mathcal{V}_{n}$ of one less dimension. Ordinarily $\mathcal{V}_{n}$ is taken to be a subspace of $\mathcal{V}_{n+1}$, but geometric algebra admits a more profound way of relating $\mathcal{V}_{n+1}$ to $\mathcal{V}_{n}$ which will be investigated here. Let $\mathcal{G}_{n+1}^{r}$ denote the subspace of all multivectors of step $r$ in $\mathcal{G}_{n+1}$. The subspace

$$
\mathcal{G}_{n+1}^{+}=\sum_{k} \mathcal{G}_{n+1}^{2 k}
$$

of all multivectors with even step is a subalgebra called the even subalgebra of $\mathcal{G}_{n+1}$. It can be identified with the geometric algebra $\mathcal{G}_{n}$ of an $n$-dimensional vector space $\mathcal{V}_{n}$, as expressed by

$$
\mathcal{G}_{n}=\mathcal{G}_{n+1}^{+} .
$$

This requires the specification of a unique relation between the vector spaces $\mathcal{V}_{n+1}=\mathcal{G}_{n+1}^{1}$ and $\mathcal{V}_{n}=\mathcal{G}_{n}^{1}$. A geometrically significant way to do this is as follows. Let $e_{0}$ and $x$ be vectors in $\mathcal{V}_{n+1}$, then, for fixed $e_{0}$ with $e_{0}^{2} \neq 0$,

$$
x e_{0}=x \cdot e_{0}+x \wedge e_{0}
$$

is a linear mapping of $\mathcal{V}_{n+1}$ into $\mathcal{G}_{n+1}^{0}+\mathcal{G}_{n+1}^{2}$, which defines a linear correspondence between $\mathcal{V}_{n+1}$ and $\mathcal{G}_{n}^{0}+\mathcal{G}_{n}^{1}$ by the identification $\mathcal{G}_{n+1}^{0}=\mathcal{G}_{n}^{0}$ and

$$
\mathcal{V}_{n}=\mathcal{G}_{n}^{1}=\left\{x \wedge e_{0}\right\}
$$

This gives $\mathcal{V}_{n}$ a projective interpretation as the pencil of all lines through the point $e_{0}$. Moreover, it determines a split of $\mathcal{G}_{n+1}^{2}$ into a direct sum,

$$
\mathcal{G}_{n+1}^{2}=\mathcal{G}_{n}^{1}+\mathcal{G}_{n}^{2}
$$

where, in accordance with $(4.4) \mathcal{G}_{n}^{1}$ consists of all bivectors in $\mathcal{G}_{n+1}^{2}$ which anticommute with $e_{0}$ while $\mathcal{G}_{n}^{2}$ consists of the bivectors which commute with $e_{0}$. The split of any bivector $F$ in $\mathcal{G}_{n+1}^{2}$ is thus given by

$$
F=F e_{0}^{-1} e_{0}=\left(F \cdot e_{0}^{-1}\right) e_{0}+\left(F \wedge e_{0}^{-1}\right) e_{0},
$$

where

$$
\left(F \cdot e_{0}^{-1}\right) e_{0}=\left(F \cdot e_{0}^{-1}\right) \wedge e_{0}
$$

is in $\mathcal{G}_{n}^{1}=\mathcal{V}_{n}$ and

$$
\left(F \wedge e_{0}^{-1}\right) e_{0}=\left(F \wedge e_{0}^{-1}\right) \cdot e_{0}
$$


is in $\mathcal{G}_{n}^{2}$.

The linear mapping (4.3) $\mathcal{V}_{n+1}$ into $\mathcal{G}_{n}$ along with the identification of $\mathcal{G}_{n+1}^{+}$with $\mathcal{G}_{n}$ according to (4.4) and (4.5) will be called a projective split of $\mathcal{V}_{n+1}$, or if you will, of $\mathcal{G}_{n+1}$. In view of its geometrical significance, the projective split can be regarded as a canonical relation between geometric algebras of different dimension. Indeed, the underlying geometrical idea played a crucial role in Clifford's original construction of geometric algebras [5]. However, Clifford's motivating ideas have been largely ignored in subsequent mathematical applications of Clifford algebras. The projective split idea was first explicitly formulated and applied to physics in [6]. Accordingly, flat spacetime is represented by a vector space $\mathcal{V}_{4}$ with Minkowski metric, and its associated geometric algebra $\mathcal{G}_{4}$ is called the spacetime algebra. The 'splitting vector' $e_{0}$ is taken to be the timelike vector of some inertial frame, and it determines a projective split of $\mathcal{V}_{4}$ and $\mathcal{G}_{4}$ into space and time components. This is called a spacetime split. Each inertial system determines a unique spacetime split. This relates the invariant properties of objects in spacetime to their 'observable' representations in inertial systems. For example, an electromagnetic field is represented by an invariant bivector-valued function $F=F(x)$ on $\mathcal{V}_{4}$. In that case, the split (4.6) describes the framedependent splitting of $F$ into electric and magnetic components. The spacetime split is thus a fundamental relation in physics, though its projective character usually goes unrecognized.

The projective split (4.3) can be put in the form

$$
x e_{0}=x_{0}(1+\mathbf{x}),
$$

where $x_{0}=x \cdot e_{0}$ and boldface is used to denote a vector

$$
\mathbf{x}=\frac{x \wedge e_{0}}{x \cdot e_{0}}
$$

in $\mathcal{V}_{n}$. Clearly $x \wedge e_{0}$ and $x \cdot e_{0}$ or just $x$ relative to $e_{0}$ amounts to a representation of the 'point' $\mathrm{x}$ by 'homogeneous coordinates'.

For 'points' $a, b$ in $\mathcal{V}_{n+1}$, the projective split (4.7) relates products in $\mathcal{G}_{n+1}$ to products in $\mathcal{G}_{n}$; thus,

$$
\begin{aligned}
a b & =\frac{\left(a e_{0}\right)\left(e_{0} b\right)}{e_{0}^{2}}=\frac{a_{0} b_{0}}{e_{0}^{2}}(1+\mathbf{a})(1+\mathbf{b}) \\
& =\frac{a_{0} b_{0}}{e_{0}^{2}}(1-\mathbf{a} \cdot \mathbf{b}+\mathbf{a}-\mathbf{b}+\mathbf{b} \wedge \mathbf{a}) .
\end{aligned}
$$

Separating inner and outer products in $\mathcal{G}_{n+1}$ we obtain

$$
a \cdot b=\frac{a_{0} b_{0}}{e_{0}^{2}}(1-\mathbf{a} \cdot \mathbf{b})
$$

and

$$
\begin{aligned}
a \wedge b & =\frac{a_{0} b_{0}}{e_{0}^{2}}(\mathbf{a}-\mathbf{b}+\mathbf{a} \wedge \mathbf{b}) \\
& =\frac{a_{0} b_{0}}{e_{0}^{2}}\left(\mathbf{a}-\mathbf{b}+\frac{1}{2}(\mathbf{a}+\mathbf{b}) \wedge(\mathbf{a}-\mathbf{b})\right) .
\end{aligned}
$$


It is important to distinguish inner and outer products in $\mathcal{G}_{n+1}$ from inner and outer products in $\mathcal{G}_{n}$. The distinction is made here by using boldface for vectors in $\mathcal{G}_{n}$.

Equation (4.10) relates two different forms for the 'Plucker coordinates' of a line passing through points $a$ and $b$. It tells us that $a \wedge b$ represents a line in $\mathcal{V}_{n}$ with direction $\mathbf{a}-\mathbf{b}$ passing through the point $\frac{1}{2}(\mathbf{a}+\mathbf{b})$. We can put (4.10) in the form

$$
a \wedge b=\frac{a_{0} b_{0}}{e_{0}^{2}}(1+\mathbf{d})(\mathbf{a}-\mathbf{b}),
$$

where

$$
\mathbf{d}=(\mathbf{b} \wedge \mathbf{a}) \cdot(\mathbf{a}-\mathbf{b})^{-1}
$$

can be regarded as the 'directed distance' from the origin to the line. For three distinct points $a, b, c$ on the same line, we have $a \wedge b \wedge c=0$, and application of (4.11) yields the invariant ratio

$$
\frac{a \wedge c}{b \wedge c}=\frac{a_{0}}{b_{0}}\left(\frac{\mathbf{a}-\mathbf{c}}{\mathbf{b}-\mathbf{c}}\right)
$$

This is a 'projective invariant' in two senses: It is independent of the chosen projective split, and it is invariant under collineations. Thus, for a collineation $f$ the linearity of the outermorphism immediately implies

$$
\frac{f(a \wedge c)}{\underline{f}(b \wedge c)}=\frac{a \wedge c}{b \wedge c} .
$$

The interval ratio $(\mathbf{a}-\mathbf{c}) /(\mathbf{b}-\mathbf{c})$ is not a projective invariant, but it is an affine invariant because $a_{0} / b_{0}$ is (see below). The classical cross-ratio for four distinct points $a, b, c, d$ on a line is given by

$$
\left(\frac{a \wedge c}{b \wedge c}\right)\left(\frac{b \wedge d}{a \wedge d}\right)=\left(\frac{\mathbf{a}-\mathbf{c}}{\mathbf{b}-\mathbf{c}}\right)\left(\frac{\mathbf{b}-\mathbf{d}}{\mathbf{a}-\mathbf{d}}\right) .
$$

This is a projective invariant in both senses mentioned above. Using (4.15), all the wellknown implications of the cross-ratio are easily derived. The considerable advantage of using geometric algebra here should be obvious. The division in (4.15) is well-defined without abuse of notation. This is an important example of a projective invariant which is not so simply expressed in terms of meet and join products alone.

The essential relation between affine and projective geometry is a projective split with respect to a preferred vector $e_{0}$. The equation

$$
x \cdot e_{0}=\lambda
$$

determines a 1-parameter family of hyperplanes in $\mathcal{V}_{n+1}$ with normal $e_{0}$. The affine group of $\mathcal{V}_{n}$ is the group of collineations on $\mathcal{V}_{n+1}$ which leaves the projective split by $e_{0}$ and, hence, the hyperplane equation (4.16), invariant. Thus, every affine transformation $f$ satisfies the equation

$$
e_{0} \cdot(\underline{f} x)=x \cdot\left(\bar{f} e_{0}\right)=x \cdot e_{0} .
$$

This is equivalent to the condition that $e_{0}$ is a fixed point of the adjoint transformation $\bar{f}$ :

$$
\bar{f} e_{0}=e_{0} .
$$


(Henceforth, the underbar and overbar notations will be used to denote linear operators as well as their outermorphisms.) by

Of special interest in affine geometry is the translation operator $\underline{T}_{a}$ defined for $a \cdot e_{0}=0$

$$
\underline{T}_{a} x=x+a x \cdot e_{0}=x+e_{0} \cdot(x \wedge a) .
$$

Its adjoint transformation is

$$
\bar{T}_{a} x=x+e_{0}(a \cdot x)=x+a \cdot\left(x \wedge e_{0}\right),
$$

which obviously satisfies the $e_{0}$ invariance condition (4.18). The translation operator induces an outermorphism of lines in $\mathcal{P}_{n}$ :

$$
\begin{aligned}
\bar{T}_{a}(x \wedge y) & =x \wedge y+a \wedge\left(y x \cdot e_{0}-x y \cdot e_{0}\right) \\
& =x \wedge y+(x \wedge y \wedge a) \cdot e_{0}
\end{aligned}
$$

For $k$ points, this generalizes to

$$
\bar{T}_{a}\left(x_{1} \wedge \cdots \wedge x_{k}\right)=x_{1} \wedge \cdots \wedge x_{k}+(-1)^{k}\left(x_{1} \wedge \cdots \wedge x_{k} \wedge a\right) \cdot e_{0} .
$$

This applies to all vectors in $\mathcal{V}_{n+1}$ whether or not they 'lie' on a single invariant hyperplane.

The linear transformation (4.19) is actually a shear on $\mathcal{V}_{n+1}$; however, it projects to a translation on $\mathcal{V}_{n}$ To show that explicitly, write $x^{\prime}=\bar{T}_{a} x$ and note that $x^{\prime} \cdot e_{0}=x \cdot e_{0}$, so the projective split (4.8) gives the translation

$$
\mathbf{x}^{\prime}=\mathbf{x}+\mathbf{a}
$$

By applying the outermorphism (4.20) to $x \wedge e_{0}$, we ascertain the important fact that the projective split is not preserved by the outermorphism.

The advantage of the projective split for affine geometry is precisely the advantage of homogeneous coordinates, namely, it reduces translations to linear transformations. In the next section we show how geometric algebra helps with a further simplification, reducing translations to rotations.

The projective split has important implications for metric geometry as well as affine geometry. For metrical applications we restrict the scalars to real numbers and we must take signature into account. We write

$$
\mathcal{V}_{n}=\mathcal{V}(p, q)
$$

when we wish to indicate that the vector space $\mathcal{V}_{n}$ has the signature $(p, q)$ imposed by its associated geometric algebra $\mathcal{G}_{n}=\mathcal{G}(p, q)$.

When the signature is taken into account in a split by $e_{0}$, the split relation (4.2) takes the more specific form

$$
\mathcal{G}(p, q)=\mathcal{G}^{+}(q+1, p) \quad \text { when } \quad e_{0}^{2}>0
$$

or

$$
\mathcal{G}(p, q)=\mathcal{G}^{+}(p, q+1) \quad \text { when } \quad e_{0}^{2}<0
$$


To characterize orthogonal transformations, it is convenient to follow [3] and introduce the concept of 'versor.' A versor $R$ in $\mathcal{G}(p, q)$ is any element which can be factored into a geometric product

$$
R=\mathbf{v}_{1} \mathbf{v}_{2} \ldots \mathbf{v}_{k}
$$

of unit vectors $\mathbf{v}_{i}$ in $\mathcal{V}(p, q)$. The factorization (4.26) is by no means unique, but there is always one with $k \leq n$. The multivector $R$ is even or odd according to whether $k$ is even or odd. This determines its parity under the main involution:

$$
R^{*}=(-1)^{k} R
$$

An even versor $R=R^{*}$ is called a rotor.

The versors in $\mathcal{G}(p, q)$ form a multiplicative group $\operatorname{Pin}(p, q)$ commonly called a 'pin group' [7]. The rotors comprise a subgroupSpin $(p, q)$ called a 'spin group'.

Now we can simply state what may be regarded as the fundamental theorem of metric geometry: Every orthogonal transformation $\underline{R}$ on $\mathcal{V}(p, q)$ can be written in the canonical form

$$
\underline{R} \mathbf{x}=R^{*} \mathbf{x} R^{-1}=R \mathbf{x}\left(R^{*}\right)^{-1},
$$

where the underbar distinguishes the linear operator $\underline{R}$ from its corresponding versor $R$. This is called a rotation if $R=R^{*}$ is a rotor.

A remark on terminology is in order. The word 'versor' comes from Clifford [5] who got it from Hamilton. Clifford explains that it is derived from the word 'reverse' in the expression 'reverse direction'. The term is employed here with the same motivation, but it is defined differently. According to (4.26), the most elementary versor is a vector $R=\mathbf{v}$, in which case (4.28) takes the form

$$
\underline{V} \mathbf{x}=-\mathbf{v x v}^{-1} .
$$

This represents a reflection $\underline{V}$ in a hyperplane with normal $\mathbf{v}$; its net effect is simply to reverse the direction of vectors collinear with $v$. Thus, each unit vector $\mathbf{v}$ can be interpreted as an operator which 'reverses' direction; whence 'versor'. From this it should be clear that the factorization (4.26) corresponds to the Cartan-Dieudonné theorem that every orthogonal transformation of $\mathcal{V}_{n}$ can be expressed as a product of at most $n$ symmetries.

The main fact of interest here is that by a projective split with respect to $e_{0} \operatorname{Spin}(p, q)$ is related to $\operatorname{Spin}(q+1, p)$ if $e_{0}^{2}=1$ or to $\operatorname{Spin}(p, q+1)$ if $e_{0}^{2}=-1$. Specifically, every rotor in $\mathcal{G}_{n+1}$ can be factored into a product

$$
S=S_{0} R
$$

where $R$ is a rotor in $\mathcal{G}_{n}$ and there exists a vector $u$ in $\mathcal{V}_{n+1}$ such that

$$
S_{0}=u e_{0} .
$$

The vector $u$ is determined by noting that $S$ determines a rotation of $e_{0}$ into a vector

$$
v=S e_{0} S^{-1}
$$

and requiring that $S_{0}$ also rotates $e_{0}$ into $v$. Then we have

$$
v=S_{0} e_{0} S_{0}^{-1}=S_{0}^{2} e_{0}^{-1},
$$


where the last step follows from assuming that $S_{0}$ has the form (4.30b). Solving for $S_{0}$ we get

$$
S_{0}=\left(v e_{0}\right)^{1 / 2}=\frac{\left(v+e_{0}\right) e_{0}}{\left|v+e_{0}\right|}=\frac{v\left(v+e_{0}\right)}{\left|v+e_{0}\right|} .
$$

Thus, $u=\left(v+e_{0}\right)\left|v+e_{0}\right|^{-1}$ is a unit vector 'halfway between' $v$ and $e_{0}$. Now $R$ is given by

$$
R=S_{0}^{-1} S
$$

and it follows that

$$
R e_{0} R^{-1}=e_{0}
$$

This implies that $R$ is in $\operatorname{Spin}(p, q)$ as claimed.

Since (4.30b) has the form of the projective split (4.3), we can regard (4.30a) as a projective split of the spin group. For the spacetime algebra $\mathcal{G}(1,3)$, Ref. [5] derives (4.30a) as a spacetime split of a Lorentz transformation into a boost (or pure Lorentz transformation) and a spatial rotation. This has many important physical applications. Mathematicians have also used the split (4.30a) to study the transitivity of spin groups on spheres (e.g. Chap. 21 of [6]) without, however, recognizing its general connection with projective geometry.

\section{Conformal and Metric Geometry}

We have seen that the projective split by a vector determines a geometrically significant relation between the geometric algebras $\mathcal{G}_{n}$ and $\mathcal{G}_{n+1}$. This section shows that a split by a bivector determines an equally significant relation between $\mathcal{G}_{n}$ and $\mathcal{G}_{n+2}$. Let $e_{0}$ be a fixed unit 2-blade in $\mathcal{G}_{n+2}^{2}$ and let $x$ be a generic vector in $\mathcal{V}_{n+1}=\mathcal{G}_{n+2}^{1}$. A linear 'split' of $\mathcal{V}_{n+2}$ into vector spaces $\mathcal{V}_{2}$ and $\mathcal{V}_{n}$ is determined by the equation

$$
x e_{0}=x \cdot e_{0}+x \wedge e_{0}=x_{0}+\rho \mathbf{x},
$$

where

$$
\mathcal{V}_{2}=\left\{x_{0}=x \cdot e_{0}=-e_{0} \cdot x\right\}
$$

and

$$
\mathcal{V}_{n}=\left\{\rho \mathbf{x}=x \wedge e_{0}=e_{0} \wedge x\right\}
$$

The significance of the scale factor $\rho$ will be discussed later. The vector space $\mathcal{V}_{2}$ generates a geometric algebra $\mathcal{G}_{2}$ with pseudoscalar $e_{0}$, and $\mathcal{V}_{n}$ generates a geometric algebra $\mathcal{G}_{n}$. We have the commutative relations

$$
\begin{aligned}
& x_{0} \mathbf{x}=\mathbf{x} x_{0}, \\
& e_{0} \mathbf{x}=\mathbf{x} e_{0} .
\end{aligned}
$$

Hence, all elements of $\mathcal{G}_{2}$ commute with the elements of $\mathcal{G}_{n}$, and we can express $\mathcal{G}_{n+2}$ as the Kronecker product

$$
\mathcal{G}_{n+2}=\mathcal{G}_{n} \otimes \mathcal{G}_{2}
$$


This is the fundamental multiplicative decomposition theorem for geometric algebras. Equation (5.5) has been treated previously only as a formal algebraic relation. The present approach gives it a geometric meaning by developing it as split by a bivector. Note that the vector space $\mathcal{V}_{n}$ defined by (5.3) has a projective interpretation: The 2-blade $e_{0}$ represents a fixed line and $\mathrm{x}=x \wedge e_{0}$ represents a plane containing that line; therefore $\mathcal{V}_{n}$ represents a pencil of planes intersecting in a common line. We will not pursue the implications of this fact for projective geometry. We will concentrate on metrical implications.

The decomposition theorem (5.5) was proved in Clifford's original article [5] for antiEuclidean signature. The signature does not play a crucial role in the proof, as the above approach shows. However, when the theorem is used to classify geometric algebras by multiplicative structure, the signature is important, as has been emphasized recently by several authors $([8],[9],[10])$. From the above, we find that (5.5) separates into three different cases depending on the signature of $\mathcal{G}_{2}$ :

$$
\begin{aligned}
& \mathcal{G}(p+1, q+1)=\mathcal{G}(p, q) \otimes \mathcal{G}(1,1), \\
& \mathcal{G}(q+2, p)=\mathcal{G}(p, q) \otimes \mathcal{G}(2,0), \\
& \mathcal{G}(q, p+2)=\mathcal{G}(p, q) \otimes \mathcal{G}(0,2) .
\end{aligned}
$$

Note that $e_{0}^{2}=1$ in (5.6) and $e_{0}^{2}=-1$ in (5.7) and (5.8); this determines the relative places of $p$ and $q$ on the two sides of the equations. The algebras $\mathcal{G}(1,1)$ and $\mathcal{G}(2,0)$ are isomorphic, though not geometrically equivalent, and both are not isomorphic to $\mathcal{G}(0,2)$. There follows a host of theorems about the algebraic equivalence of algebras with different signature [8-10], but that will not concern us here. We will be concerned only with the geometric significance of (5.6), which we abbreviate by

$$
\mathcal{G}_{n+2}=\mathcal{G}_{n} \otimes \mathcal{G}(1,1)
$$

when the particular signature $(p, q)$ is not a matter of concern. Anticipating the results to follow, let us refer to this as a conformal split of $\mathcal{G}_{n+2}$ by the unit 2-blade $e_{0}$.

From our previous considerations, we know that every orthogonal transformation of $\mathcal{V}_{n+2}=\mathcal{V}(p+1, q+1)$ has the canonical form

$$
x^{\prime}=G x\left(G^{*}\right)^{-1},
$$

where $G$ is an element of the versor group $\operatorname{Pin}(p+1, q+1)$. The theorem of central interest in the remainder of this section is that the orthogonal group $\mathrm{O}(p+1, q+1)$ is isomorphic to the complete conformal group $\mathrm{C}(p, q)$ on $\mathcal{V}_{n}=\mathcal{V}(p, q)$; therefore $\operatorname{Pin}(p+1, q+1)$ is the spin representation of $\mathrm{C}(p, q)$. We will use the conformal split to prove this theorem and analyze the structure of the conformal group and its spin representation in detail.

Representations of conformal groups in Clifford algebras have been discussed by many authors, notably Angles [11], Lounesto and Latvamaa [12], Ahlfors [13], Maks [14], and Crumeyrolle [15]. The present study aims to show that the conformal split idea simplifies algebraic manipulations, clarifies geometric meanings, and reveals connections among alternative approaches. A complete, efficient and systematic treatment of the conformal group should be helpful in applications to physics. The most thorough discussion of the physical significance of the conformal group for spacetime has been given by Kastrup [16].

Our plan of study for the remainder of this Section is as follows. First, we analyze the algebra $\mathcal{G}(1,1)$ and $\operatorname{Pin}(1,1)$ thoroughly. Though the results are well-known, the analysis is 
necessary to establish notation for subsequent application. Next, we establish homogeneous coordinates for $\mathcal{V}_{n}$ in $\mathcal{G}_{n+2}$ and the general relation of the Pin group to the conformal group. Finally, we determine a canonical factorization of the groups into elementary generators.

\subsection{STRUCTURE OF $\mathcal{G}(1,1) \operatorname{AND} \operatorname{Pin}(1,1)$}

An orthonormal basis $\left\{e_{1}, e_{2}\right\}$ for the vector space $\mathcal{V}_{2}=\mathcal{V}(1,1)$ is defined by the properties

$$
e_{1}^{2}=1, \quad e_{1}^{2}=-1, \quad e_{1} \cdot e_{2}=0,
$$

A null basis $\left\{e_{+}, e_{-}\right\}$for $\mathcal{V}(1,1)$ is defined by

$$
e_{ \pm}=\frac{1}{2}\left(e_{1} \pm e_{2}\right)
$$

It has the properties

$$
e_{+}^{2}=e_{-}^{2}=0
$$

and

$$
e_{+} e_{-}=\frac{1}{2}\left(1+e_{0}\right)
$$

This latter relation separates into

$$
2 e_{+} \cdot e_{-}=1=2 e_{+} \cdot e_{1}=2 e_{-} \cdot e_{2}
$$

and

$$
2 e_{+} \wedge e_{-}=e_{0}=e_{2} e_{1}
$$

The bivector $e_{0}$ is the unit pseudoscalar for $\mathcal{V}_{2}$. It has the important properties

$$
\begin{gathered}
e_{0}^{2}=1, \\
e_{0} e_{ \pm}= \pm e_{ \pm}=-e_{ \pm} e_{0} .
\end{gathered}
$$

The null basis has the advantage of being unique (up to a scale factor), since it characterizes intrinsic structure of $\mathcal{V}(1,1)$, namely its 'isotropic cone.' For this reason, we use the null basis whenever feasible. However, null vectors are not elements of the versor group, so we use $e_{1}$ and $e_{2}$ to represent reflections.

For comparison with the literature, it should be understood that $\mathcal{G}(1,1)$ is isomorphic to the algebra $L_{2}(R)$ of real $2 \times 2$ matrices, that is,

$$
\mathcal{G}(1,1) \simeq L_{2}(R)
$$

To make the isomorphism explicit, we write a generic multivector $M$ in $\mathcal{G}(1,1)$ in the expanded form

$$
M=\frac{1}{2}\left[A\left(1+e_{0}\right)+B\left(e_{1}+e_{2}\right)+C\left(e_{1}-e_{2}\right)+D\left(1-e_{0}\right)\right],
$$

where $A, B, C, D$ are scalars. The basis elements have the matrix representation

$$
\left[e_{1}\right]=\left[\begin{array}{ll}
0 & 1 \\
1 & 0
\end{array}\right], \quad\left[e_{2}\right]=\left[\begin{array}{cc}
0 & 1 \\
-1 & 0
\end{array}\right], \quad\left[e_{0}\right]=\left[\begin{array}{cc}
1 & 0 \\
0 & -1
\end{array}\right] .
$$


Consequently (5.20) has the matrix representation

$$
[M]=\left[\begin{array}{ll}
A & B \\
C & D
\end{array}\right] .
$$

The matrix representation preserves the geometric product, that is

$$
[M N]=[M][N] \text {. }
$$

The conjugate $\tilde{M}$ can be defined by

$$
\widetilde{M}=\left(M^{*}\right)^{\dagger},
$$

where the dagger denotes reversion in $\mathcal{G}(1,1)$. The involute is given by

$$
M^{*}=e_{0} M e_{0} .
$$

It is readily verified that

$$
\operatorname{det}[M]=\tilde{M} M .
$$

Therefore, we have the isomorphisms

$$
\begin{aligned}
& \{M \mid M \widetilde{M} \neq 0\} \simeq \mathrm{GL}_{2}(R), \\
& \{M \mid M \widetilde{M}=1\} \simeq \mathrm{SL}_{2}(R),
\end{aligned}
$$

to the general linear and special linear groups.

The group (5.28) is a 3-parameter group. Its structure is revealed by the following canonical decomposition: If $M \widetilde{M}=1$, then there are values of scalar parameters $\lambda, \alpha, \beta$ in the interval $[-\infty, \infty]$ such that (modulo \pm 1 )

$$
M=K_{\alpha} T_{\beta} D_{\lambda},
$$

where

$$
\begin{aligned}
& K_{\alpha}=e^{\alpha e_{-}}=1+\alpha e_{-}, \\
& T_{\beta}=e^{\beta e_{+}}=1+\beta e_{+}, \\
& D_{\lambda}=\frac{1}{2}\left(1+e_{0}\right) \lambda+\frac{1}{2}\left(1-e_{0}\right) \lambda^{-1}=e^{\phi e_{0}}
\end{aligned}
$$

for $\lambda=e^{\phi}$. An alternative to (5.29) is the so-called Iwasawa decomposition

$$
M=V_{\alpha} T_{\beta} D_{\lambda}
$$

where, for $\alpha=\cosh \theta$,

$$
V_{\alpha}=\alpha+e_{1}\left(1-\alpha^{2}\right)^{1 / 2}=e^{\theta e_{1}} .
$$

The Iwasawa decomposition has been much used in the theory of $\mathrm{SL}_{2}(R)$ group representations [17]. However, it will be seen that (5.29) is more significant in the present context, because $e_{+}, e_{-}$and $e_{0}$ are geometrically unique generators, whereas $e_{1}$ is not. 
For the matrix representation of (5.29), we get

$$
\begin{aligned}
{[M] } & =\left[K_{\alpha}\right]\left[T_{\beta}\right]\left[D_{\lambda}\right] \\
& =\left[\begin{array}{ll}
1 & 0 \\
\alpha & 1
\end{array}\right]\left[\begin{array}{cc}
1 & \beta \\
0 & 1
\end{array}\right]\left[\begin{array}{cc}
\lambda & 0 \\
0 & \lambda^{-1}
\end{array}\right] \\
& =\left[\begin{array}{cc}
\lambda & \beta \lambda^{-1} \\
\alpha \lambda & (1+\alpha \beta) \lambda^{-1}
\end{array}\right] .
\end{aligned}
$$

For $V_{\lambda}$ given by (5.34) with $\lambda=\alpha$ this gives the matrix representation

$$
\left[V_{\lambda}\right]=\left[K_{\alpha} T_{\beta} D_{\lambda}\right]=\left[\begin{array}{cc}
\lambda & \beta \lambda^{-1} \\
\alpha \lambda & \lambda
\end{array}\right],
$$

where $\lambda^{2}=\alpha / \beta=(1+\alpha \beta)$. This shows the equivalence of (5.29) and (5.33).

An important feature of (5.29) is that it separates distinct and unique 1-parameter subgroups $K_{\alpha}, T_{\beta}, D_{\lambda}$, as well as such 2-parameter subgroups as $T_{\beta} D_{\lambda}$. Of these, it will be seen below that only $D_{\lambda}$ belongs to $\operatorname{Pin}(p+1, q+1)$; however, $K_{\alpha}$ and $T_{\beta}$ play a role in connecting $\operatorname{Pin}(1,1)$ to $\operatorname{Pin}(p, q)$.

The spin group of $\mathcal{G}(1,1)$ consists of

$$
\operatorname{Spin}(1,1)=\left\{e_{0}, D_{\lambda}\right\}
$$

where $D_{\lambda}$ is given by (5.32). The rotor $D_{\lambda}$ represents the 1-parameter family of 'Lorentz transformations' of $\mathcal{V}_{2}$. By (5.18),

$$
D_{\lambda} e_{ \pm} D_{\lambda}^{-1}=D_{\lambda}^{2} e_{ \pm}=\lambda^{ \pm 2} e_{ \pm}
$$

As noted in (5.25), the rotor $e_{0}$ represents the involution

$$
e_{0} e_{ \pm} e_{0}=-e_{ \pm}=e_{ \pm}^{*}
$$

The rest of $\operatorname{Pin}(1,1)$ is generated by $e_{1}$, which represents the reflection

$$
e_{1} e_{ \pm} e_{1}=e_{\mp}
$$

The reflection represented by $e_{2}=e_{0} e_{1}$ is a composite of (5.39) and (5.40), and all other reflections of $\mathcal{V}(1,1)$ cam be obtained by composites with rotations.

\subsection{HOMOGENEOUS COORDINATES AND THE CONFORMAL SPLIT OF $\operatorname{Pin}(p+1, q+1)$}

Like the projective split, the conformal split (5.1) can be regarded as assigning homogeneous coordinates to the 'points' in $\mathcal{V}_{n}$. There are two extra degrees of freedom in $\mathcal{V}_{n+2}$, but one of them can be eliminated by requiring $x^{2}=0$ for points $x$ in $\mathcal{V}_{n+2}$ representing points in $\mathcal{V}_{n}$. Then the conformal split (5.1) gives us

$$
x^{2}=\left(x e_{0}\right)\left(e_{0} x\right)=\left(x \wedge e_{0}+x \cdot e_{0}\right)\left(x \wedge e_{0}-x \cdot e_{0}\right)=0,
$$


whence

$$
\left(x \cdot e_{0}\right)^{2}=\left(x \wedge e_{0}\right)^{2}=\rho^{2} \mathbf{x}^{2} .
$$

Unique homogeneous coordinates for $x$ will be determined by a suitable choice of scale factor $\rho$. From (5.16) we get

$$
\frac{1}{2} x \cdot e_{0}=x \cdot\left(e_{+} \wedge e_{-}\right)=\left(x \cdot e_{+}\right) e_{-}-\left(x \cdot e_{-}\right) e_{+} .
$$

Squaring and using (5.15) and (5.41), we obtain

$$
\left(x \cdot e_{0}\right)^{2}=-4 x \cdot e_{+} x \cdot e_{-}=\rho^{2} \mathbf{x}^{2} .
$$

Evidently, the simplest choice of scale factor is

$$
\rho=2 x \cdot e_{+},
$$

for then

$$
x \cdot e_{-}=-\mathbf{x}^{2} x \cdot e_{+} .
$$

Inserting (5.42) and (5.43) into (5.1), we get the conformal split in the form

$$
x e_{0}=2 x \cdot e_{+}\left(e_{-}+\mathbf{x}^{2} e_{+}+2 \mathbf{x}\right) \equiv \rho X,
$$

where

$$
\mathbf{x}=\frac{x \wedge e_{0}}{x \cdot e_{+}}
$$

expresses $\mathbf{x}$ in terms of 'homogeneous coordinates' $x \cdot e_{+}$and $x \wedge e_{0}$. It is of interest to note, using (5.20) and (5.21), that (5.44) has the $2 \times 2$ matrix representation

$$
[X]=\left[\begin{array}{cc}
\mathbf{x} & \mathbf{x}^{2} \\
a & \mathbf{x}
\end{array}\right]
$$

This particular kind of split into homogeneous coordinates should be generally useful in that part of projective geometry dealing with a null quadratic form ('Cayley's absolute'). We show below that it is very useful in conformal and metric affine geometry.

To make the conformal split of the orthogonal transformation (5.10) explicit, we introduce the abbreviation

$$
\widehat{G}=e_{0}\left(G^{*}\right)^{-1}
$$

and write $(5.10)$ in the form

$$
G X \widehat{G}=\sigma X^{\prime} .
$$

For $x^{2}=0$, we can use (5.44) to put this in the explicit form

$$
G\left(e_{-}+\mathbf{x}^{2} e_{+}+\mathbf{x}\right) \widehat{G}=\sigma\left[e_{-}+[g(\mathbf{x})]^{2} e_{+}+g(\mathbf{x})\right]
$$

where

$$
\mathbf{x}^{\prime}=g(\mathbf{x})
$$


is a transformation on $\mathcal{V}_{n}$ involving a scale change

$$
\sigma=\sigma_{g}(\mathbf{x})=\frac{x^{\prime} \cdot e_{+}}{x^{\prime} \cdot e_{+}}
$$

Equation (5.49) is the main theorem relating the group $\operatorname{Pin}(p+1, q+1)=\{G\}$ to the conformal group $\mathrm{C}(p, q)=\{g\}$. It has been studied by Angles [11], but without the conformal split to simplify the analysis.

To prove the main theorem, it is necessary to establish that every function $g$ determined by $G$ in (5.49) is conformal and that every conformal transformation can be obtained in this way. Instead of approaching the proof directly, we achieve it as a byproduct of analyzing the structural relationships between the two groups in detail. The analysis can be carried out in two complementary ways.

One way is to note, by considering (5.20), that every multivector $G$ in $\mathcal{G}_{n+2}$ can be expressed in the form

$$
G=A e_{+} e_{1}+B e_{+}+C e_{-}+D e_{-} e_{1},
$$

where the 'coefficients' $A, B, C, D$ are in $\mathcal{G}_{n}$. The operations of reversion and involution yield

$$
G^{\dagger}=D^{\dagger} e_{+} e_{1}+B^{\dagger} e_{+}+C^{\dagger} e_{-}+A^{\dagger} e_{-} e_{1}
$$

and

$$
G^{*}=A^{*} e_{+} e_{1}-B^{*} e_{+}-C^{*} e_{-}+A^{*} e_{-} e_{1} .
$$

Equation (5.52a) differs from (5.20) only in the replacement of scalars by elements of $\mathcal{G}_{n}$. Since the elements of $\mathcal{G}(1,1)$ commute with those of $\mathcal{G}_{n}$, this replacement applies as well to the matrix representation (5.22). Thus, we have the isomorphism

$$
\mathcal{G}_{n+2}=\mathcal{G}_{n} \otimes \mathcal{G}(1,1) \simeq L_{2}\left(\mathcal{G}_{n}\right)
$$

where the right side denotes the algebra of $2 \times 2$ matrices with elements in $\mathcal{G}_{n}$. Accordingly, for the matrix representations of $(5.52 \mathrm{a}, \mathrm{b}, \mathrm{c})$ we have

$$
[G]=\left[\begin{array}{cc}
A & B \\
C & D
\end{array}\right], \quad\left[G^{\dagger}\right]=\left[\begin{array}{cc}
D^{\dagger} & B^{\dagger} \\
C^{\dagger} & A^{\dagger}
\end{array}\right], \quad\left[G^{*}\right]=\left[\begin{array}{cc}
A^{*} & -B^{*} \\
-C^{*} & D^{*}
\end{array}\right] .
$$

The matrix representation has the advantage of compactness and easy multiplication, but the disadvantage of suppressing the relation to vectors in $\mathcal{G}(1,1)$ and so complicating geometric interpretation. This kind of matrix representation for the conformal group has been employed by Ahlfors [13] and Maks [14].

The requirement that $G$ is an element of $\operatorname{Pin}(p+1, q+1)$ puts restrictions on the coefficients in (5.52a). First, $G$ must satisfy the versor condition

$$
G G^{\dagger}=|G|^{2}
$$

where $|G|^{2}$ is a nonvanishing scalar (possibly negative, unless the signature of $\mathcal{V}_{n}$ is Euclidean). In the matrix representation (5.54), this condition is expressed by

$$
\left[G G^{\dagger}\right]=\left[\begin{array}{ll}
A D^{\dagger}+B C^{\dagger} & A B^{\dagger}+B A^{\dagger} \\
C D^{\dagger}+D C^{\dagger} & C B^{\dagger}+D A^{\dagger}
\end{array}\right]=|G|^{2}\left[\begin{array}{ll}
1 & 0 \\
0 & 1
\end{array}\right]
$$


Second, Equation (5.10) or, equivalently, (5.49) must be satisfied. The matrix representation of $(5.47)$ can be written

$$
\widehat{G}=\left[\begin{array}{ll}
\widehat{D} & \widehat{B} \\
\widehat{C} & \widehat{A}
\end{array}\right]
$$

with the notation

$$
\widehat{A}=\left(A^{\dagger}\right)^{*}|G|^{-2} .
$$

Then (5.49) gives us the condition

$$
\begin{aligned}
{\left[G X G^{\dagger}\right] } & =\left[\begin{array}{cc}
(A \mathbf{x}+B)(\widehat{D}+\mathbf{x} \widehat{C}) & (A \mathbf{x}+B)(\widehat{B}+\mathbf{x} \widehat{A}) \\
(C \mathbf{x}+D)(\widehat{D}+\mathbf{x} \widehat{C}) & (C \mathbf{x}+D)(\widehat{B}+\mathbf{x} \widehat{A})
\end{array}\right] \\
& =\sigma\left[\begin{array}{cc}
g(\mathbf{x}) & {[g(\mathbf{x})]^{2}} \\
1 & g(\mathbf{x})
\end{array}\right]
\end{aligned}
$$

where $\sigma$ is a scalar and $g(x) \in \mathcal{V}_{n}$. From (5.56) and (5.59) we can easlly read off a list of necessary and sufficient conditions on $A, B, C, D$. In particular, from (5.59) we find that

$$
\sigma=(C \mathbf{x}+D)(\widehat{D}+\mathbf{x} \widehat{C})
$$

and $g$ can be expressed in the homographic form

$$
g(\mathbf{x})=(A \mathbf{x}+B)(C \mathbf{x}+D)^{-1}
$$

Ahlfors [13] calls this a Möbius transformation. The big advantage of the versor representation in $\mathrm{P}(p+1, q+1)$ over the elegant Möbius form for a conformal transformation is that composition of transformations is reduced to a versor product. This, in turn, simplifies proofs and applications of a whole host of theorems, such as the canonical decomposition theorem proved below.

To prove that (5.61) is indeed a conformal transformation, we compute its differential $g(\mathbf{a})=g(\mathbf{a}, \mathbf{x})$ by taking the directional derivative. Using the fact $\mathbf{a} \cdot \boldsymbol{\nabla} \mathbf{x}=\mathbf{a}$, we first compute

$$
\mathbf{a} \cdot \boldsymbol{\nabla}(C \mathbf{x}+D)^{-1}=\mathbf{a} \cdot \boldsymbol{\nabla}\left[\frac{\mathbf{x} \widehat{C}+\widehat{D}}{\sigma}\right]=\left[\frac{\mathbf{a} \widehat{C}(C \mathbf{x}+D)-\mathbf{a} \cdot \nabla \sigma}{\sigma}\right](C \mathbf{x}+D)^{-1},
$$

whence

$$
\mathbf{a} \cdot \boldsymbol{\nabla}(C \mathbf{x}+D)^{-1}=-(C \mathbf{x}+D)^{-1} C \mathbf{a}(C \mathbf{x}+D)^{-1}
$$

Now,

$$
\begin{aligned}
\mathbf{a} \cdot \boldsymbol{\nabla} g & =\left[A \mathbf{a}-(A \mathbf{x}+B)(C \mathbf{x}+D)^{-1} C \mathbf{a}\right](C \mathbf{x}+D)^{-1} \\
& =\left(\mathbf{x} C^{\dagger}+D^{\dagger}\right)^{-1}\left[\left(\mathbf{x} C^{\dagger}+D^{\dagger}\right) A-\left(\mathbf{x} A^{\dagger}+B^{\dagger}\right) C\right] \mathbf{a}(C \mathbf{x}+D)^{-1},
\end{aligned}
$$

where $g=g^{\dagger}$ has been used. Applying the special conditions on the 'coefficients', we obtain

$$
g(\mathbf{a})=\mathbf{a} \cdot \nabla g=(\mathbf{x} \widehat{C}+\hat{D})^{-1} \mathbf{a}(C \mathbf{x}+D)^{-1}
$$


This immediately yields the defining condition for a conformal transformation:

$$
[\underline{g}(\mathbf{a})] \cdot[\underline{g}(\mathbf{b})]=\frac{\mathbf{a} \cdot \mathbf{b}}{\sigma^{2}} .
$$

Thus, the proof that $g$ is conformal is complete.

Now we turn to an alternative way to analyze the group structure, exploiting the fact (4.26) that every versor element $G$ can be generated as a product of vectors in $\mathcal{V}_{n+2}$. First, we determine the explicit imbedding of $\operatorname{Pin}(p, q)$ in $\operatorname{Pin}(p+1, q+1)$ By the condition (5.2), $\mathcal{V}_{2}$ is a subspace of $\mathcal{V}_{n+2}$ and vectors $a, b$ in $\mathcal{V}_{n+2}$ are in the subspace orthogonal to $\mathcal{V}_{2}$ if $a \cdot e_{0}=b \cdot e_{0}=0$. According to (5.3), then,

$$
a=\mathbf{a} e_{0}=e_{0} \mathbf{a}
$$

and

$$
a b=\mathbf{a b},
$$

where $\mathbf{a}=a e_{0}=a \wedge e_{0}, \mathbf{b}=b \wedge e_{0}$ are vectors in $\mathcal{V}_{n}$. This determines how $\operatorname{Pin}(p, q)$ fits into $\operatorname{Pin}(p+1, q+1)$ as a subgroup. Specifically, for every versor

$$
R=\mathbf{v}_{1} \mathbf{v}_{2} \ldots \mathbf{v}_{k}
$$

in $\operatorname{Pin}(p, q)$, there are $k$ unit vectors $v_{i}=\mathbf{v}_{i} e_{0}$ in $\mathcal{V}_{n+2}$ such that

$$
\begin{aligned}
& v_{1} v_{2} \ldots v_{k}=e_{0} R \quad \text { for } k \text { odd, } \\
& v_{1} v_{2} \ldots v_{k}=R \quad \text { for } k \text { even. }
\end{aligned}
$$

From (5.43) we note that

$$
a \wedge b=\mathbf{a} \wedge \mathbf{b},
$$

which tells us that all bivectors in $\mathcal{G}_{n}$ are also bivectors in $\mathcal{G}_{n+2}$, even though (5.3) says that the vectors in $\mathcal{G}_{n}$ are 3 -vectors in $\mathcal{G}_{n+2}$. This implies that the Lie algebra of $\operatorname{Spin}(p, q)$ is contained in the Lie algebra of $\operatorname{Spin}(p+1, q+1)$, but we will not be using that important fact.

Now we are prepared to systematically classify the conformal transformations generated by vectors in $\operatorname{Pin}(p+1, q+1)$. First, we readily check that the imbedding of $\operatorname{Pin}(p, q)$ defined by (5.67a) generates the orthogonal group $\mathrm{O}(p, q)$ when employed in the main theorem (5.49). Indeed, we find $\widehat{R}=\left(R^{*}\right)^{-1}$ and

$$
g(\mathbf{x})=R \mathbf{x}\left(R^{*}\right)^{-1}
$$

as required.

\subsection{INVERSIONS, INVOLUTIONS AND DILATATIONS}

Next we classify the conformal transformations generated by $\operatorname{Pin}(1,1)$, which is also a subgroup of $\operatorname{Pin}(p+1, q+1)$. For a reflection of $\mathcal{V}_{n+2}$ generated by $e_{1}$, using (5.40) we obtain

$$
e_{1}\left(e_{-}+\mathbf{x}^{2} e_{+}+\mathbf{x}\right) e_{1}=\mathbf{x}^{2}\left(e_{-}+\mathbf{x}^{-2} e_{+}+\mathbf{x}^{-1}\right)
$$


whence

$$
g(\mathbf{x})=\mathbf{x}^{-1}=\frac{\mathbf{x}}{\mathbf{x}^{2}}
$$

Therefore, $e_{1}$ is a versor representation of inversion in $\mathcal{V}_{n}$. Of course (5.70b) is not defined along null lines $\mathbf{x}^{2}=0$. Similarly,

$$
e_{0}\left(e_{-}+\mathbf{x}^{2} e_{+}+\mathbf{x}\right) e_{0}=-\left(e_{-}+\mathbf{x}^{-2} e_{+}-\mathbf{x}\right) .
$$

Here $\sigma=-1$, and

$$
g(\mathbf{x})=-\mathbf{x} .
$$

Therefore, $e_{0}$ is a versor representation of involution in $\mathcal{V}_{n}$. Since $e_{2}=e_{0} e_{1}$, it generates the composite of $(5.70 \mathrm{~b})$ and $(5.71 \mathrm{~b})$. Therefore, $e_{2}$ represents an involutory inversion.

For $D_{\lambda}$ defined by (5.32), with the help of (5.38) we obtain

$$
D_{\lambda}\left(e_{-}+\mathbf{x}^{2} e_{+}+\mathbf{x}\right) D_{\lambda}^{-1}=\lambda^{-2}\left[e_{+}+\left(\lambda^{2} \mathbf{x}\right)^{2} e_{-}+\lambda^{2} \mathbf{x}\right]
$$

whence

$$
g(\mathbf{x})=\lambda^{2} \mathbf{x} .
$$

Therefore, $D_{\lambda}$ is the spin representation of a dilatation by $\lambda^{2}$. From (5.32) it is also readily established that every unit vector in $\mathcal{G}(1,1)$ with positive signature can be expressed in the form

$$
e=D_{\lambda} e_{1} D_{\lambda}^{-1}=e^{2 \phi e_{0}} e_{1}
$$

or the form $-e=e_{0} e e_{0}$. It follows that $\mathcal{G}(1,1)$ is a double representation of the group of inversions, involutions and dilatations on $\mathcal{V}(p, q)$.

\subsection{TRANSLATIONS, TRANSVECTIONS, AND DIVERSIONS}

Our final task is to classify the conformal transformation generated by versors with nonvanishing components in both $\mathcal{V}_{2}$ and its orthogonal complement. According to (5.1), any such vector $v$ can be written in form

$$
v=e+a,
$$

where $e=\left(v \cdot e_{0}\right) e_{0}$ and $\mathbf{a}=a e_{0}$ is in $\mathcal{V}_{n}$. Also, the versor condition

$$
v^{2}=e^{2}+\mathbf{a}^{2} \neq 0
$$

must be satisfied, but it can be scaled to any convenient value. We have two distinct cases to consider. Case I: $e^{2}=0$ implies $\mathbf{a}^{2} \neq 0$, so we can choose $e=e_{+}$or $e_{-}$. Case II: $e^{2} \neq 0$, so we can choose $e=e_{1}$ or $e_{2}$, since

$$
e+a=D_{\lambda}\left(e_{1}+a\right) D_{\lambda}^{-1}
$$

by (5.73) enables us to get the other possibilitiesby composition with dilatations.

For Case $\mathbf{I}$ let us write $v=e+a^{-1}$ and consider the rotor

$$
T_{a}=a v=1+a e_{+} .
$$


Anticipating its interpretation as the spin representation of a translation, let us refer to $T_{a}$ as a translator. By (5.18) and (5.65) we have $a e_{+}=a e_{0} e_{+}=\mathbf{a} e_{+}$, so we can write

$$
T_{\mathbf{a}}=1+\mathbf{a} e_{+}=1+e_{+} \mathbf{a}=1+a e_{+}=T_{a}
$$

showing that $T_{(a)}$ is parameterized by vectors in $\mathcal{V}_{n}$. Applied in (5.49), this yields

$$
T_{\mathbf{a}}\left(e_{-}+\mathbf{x}^{2} e_{+}+\mathbf{x}\right) T_{\mathbf{a}}=e_{-}+(\mathbf{x}+\mathbf{a})^{2} e_{+}+(\mathbf{x}+\mathbf{a}),
$$

thus determining the translation

$$
g(\mathbf{x})=\mathbf{x}+\mathbf{a},
$$

as anticipated.

The translators form a group with the properties

$$
\begin{gathered}
T_{a} T_{b}=T_{a+b} \\
T_{a}^{-1}=T_{-a}=e_{0} T_{a} e_{0} .
\end{gathered}
$$

Note that if $a^{2}=-b^{2}$, then $a+b$ is a null vector. Hence, (5.80) shows that the translator (5.78) applies to null vectors, though in that case it cannot be factored into a productof two vectors as in (5.77). However, (5.80) shows that it can be factored into a product of four vectors.

Now define

$$
K_{a}=e_{1} T_{a} e_{1}=1-a e_{-}=1+\mathbf{a} e_{-}=K_{\mathbf{a}},
$$

where (5.40) and (5.18) have been used. Thus, the interchange of $e_{+}$with $e_{-}$in (5.78) is the equivalent of an inversion followed by a translation and another inversion, so we can use (5.70a) and (5.79a) to evaluate

$$
\begin{gathered}
K_{a}\left(e_{-}+\mathbf{x}^{2} e_{+}+\mathbf{x}\right) K_{a}=\mathbf{x}^{2} e_{1}\left[e_{-}+\left(\mathbf{x}^{-1}+\mathbf{a}\right)^{2} e_{+}+\left(\mathbf{x}^{-1}+\mathbf{a}\right)\right] e_{1} \\
=\mathbf{x}^{2}\left(\mathbf{x}^{-1}+\mathbf{a}\right)^{2}\left[e_{-}+\left(\mathbf{x}^{-1}+\mathbf{a}\right)^{-2} e_{+}+\left(\mathbf{x}^{-1}+\mathbf{a}\right)^{-1}\right] .
\end{gathered}
$$

In this case,

$$
\sigma(\mathbf{x})=\mathbf{x}^{2}\left(\mathbf{x}^{-1}+\mathbf{a}\right)^{2}=1+2 \mathbf{a} \cdot \mathbf{x}+\mathbf{a}^{2} \mathbf{x}^{2}
$$

and

$$
g(\mathbf{x})=\left(\mathbf{x}^{-1}+\mathbf{a}\right)^{-1}=\mathbf{x}(1+\mathbf{a x})^{-1}=\frac{\mathbf{x}+\mathbf{a} \mathbf{x}^{2}}{\sigma(\mathbf{x})},
$$

This kind of conformal translation is called a transversion. This completes the analysis of Case I.

The analysis of Case $\mathbf{I}$ can be summarized as follows. From (5.77) we conclude that the vector $v=a^{-1} T_{a}=e_{+}+a^{-1}$ represents a translation-reflection. Similarly, $v=a^{-1} K_{a}=$ $e_{-}+a^{-1}$ represents a transvection-reflection. Turning to Case II, where $v=e_{1}+a$, we first ask whether $v$ can be generated from $e_{1}$ by a translation. Computing

$$
T_{a} e_{1} T_{a}^{-1}=e_{1}+a-a^{2} e_{+}
$$


we see that the answer is yes only if $a^{2}=0$. So we exclude $a^{2}=0$ from this case to assure that it is completely distinct from the other possibilities.

Now for Case II let us compute the conformal transformation generated by the rotor

$$
V_{a}=v e_{1}=\left(e_{1}+a\right) e_{1}=1+a e_{1}=1+\mathbf{a} e_{2} .
$$

The simplest way to do that is to write $e_{2}=e_{+}-e_{-}$and, by comparison of (5.52a) with (5.61), read off the result

$$
g(\mathbf{x})=(\mathbf{x}+\mathbf{a})(1-\mathbf{a x})^{-1} .
$$

This elementary type of conformal transformation and the fact that it is continuously connected to the identity does not seem to have been noted previously in the literature, probably because it cannot be generated by the more obvious classical conformal transformations. Let us call it a diversion.

[On the contrary, P. Lounesto has pointed out that the diversion (5.85) can be factored into the product $V_{a}=\lambda T_{a} D_{\lambda} K_{-a}$ where $\lambda^{2}=1+\mathbf{a}^{2}$.]

The effect of interchanging $e_{1}$ and $e_{2}$ in (5.85) is merely to change the sign of the first a in (5.86), but this is a new kind of diversion not reducible to (5.86). According to (5.76), we can obtain every other diversion by composition with a dilatation and its inverse. Hence, the most general form for a diversion is

$$
V_{a}=1+\mathbf{a} e,
$$

and we may call the diversion positive if $e^{2}=1$ or negative if $e^{2}=-1$.

An interesting result is obtained from the following product of two diversions:

$$
\left(1+\mathbf{a} e_{2}\right)\left(1+\mathbf{b} e_{1}\right)=(1+\mathbf{a b}) e_{+} e_{1}+(\mathbf{a}+\mathbf{b}) e_{+}+(\mathbf{b}-\mathbf{a}) e_{-}+(1-\mathbf{a b}) e_{-} e_{1} .
$$

Note that all the coefficients on the right are null if $\mathbf{a}^{2}=-\mathbf{b}^{2}$. This example was first adduced by Maks [14] as a counterexample to the common belief that every special conformal transformation can be generated by rotations, translations, inversions and dilatations [in any order] . However, we have shown above that the diversion is a more fundamental counterexample, and its existence does not require an indefinite metric. [Delete the preceding sentence.] This concludes our analysis of Case II.

\subsection{THE CONFORMAL DECOMPOSITION THEOREMS}

The main theorem (5.49) establishes the double covering of the conformal group $\mathrm{C}(p, q)$ by the versor group $\operatorname{Pin}(p+1, q+1)$. Similarly, $\operatorname{Spin}(p+1, q+1)$ is a double covering of the special conformal group $\mathrm{SC}(p, q)$, consisting of all conformal transformations continuously connected to the identity. The fundamental decomposition theorem for $\operatorname{SC}(p, q)$ can be expressed as follows: The rotor $G$ representing any combination of rotations, translations, transversions, and dilatations can [usually] be put in the canonical form

$$
G= \pm K_{\mathbf{a}} T_{\mathbf{b}} D_{\lambda} R
$$

where the factors are defined by (5.82), (5.78), (5.32) and (5.67c). [The counterexample of Maks shows that the order of factors may matter in special cases.] 
This result is easily proved with the help of the following relations to reorder and combine factors

$$
\begin{gathered}
D_{\lambda} T_{a}=T_{a^{\prime}} D_{\lambda} \quad \text { where } a^{\prime}=\lambda^{-2} a, \\
R T_{a}=T_{a^{\prime}} R \quad \text { where } a^{\prime}=R\left(R^{*}\right)^{-1} .
\end{gathered}
$$

From our preceding analysis, it follows that any element of $\operatorname{SC}(p, q)$ can be represented as a product of (5.89) with the positive and negative diversions represented by (5.87).

The most general decomposition theorem for $\mathrm{C}(p, q)$ asserts that any element can be represented in $\operatorname{Pin}(p+1, q+1)$ by versors of the form $V G, e_{0} V G, e_{1} V G$ or $e_{0} e_{1} V G$, where $V$ is a diversion and $G$ has the form (5.89), but with $R$ of the general form (5.67a) representing an orthogonal transformation.

Subgroups of the conformal group can be represented in geometric algebras of lower dimension. The group $\operatorname{Spin}(p+1, q+1)$ belongs to the even subalgebra $\mathcal{G}_{n+2}^{+}=\mathcal{G}_{n+1}$, so its action on vectors can be represented in $\mathcal{G}_{n+1}$ by a projective split. Under a projective split by $e_{2}$, Equation (5.44) gives

$$
x e_{2}=x e_{0} e_{1}=\frac{1}{2}\left(1-e_{0}\right)+\frac{1}{2}\left(1+e_{0}\right) \mathbf{x}_{1}^{2}+\mathbf{x}_{1},
$$

where the scale has been fixed by setting $x \cdot e_{+}=1$, and the projected point in $\mathcal{V}_{n}$ is now represented by

$$
\mathbf{x}_{1}=\left(x \wedge e_{0}\right) \cdot e_{1}=x \wedge e_{1}+\left(x \cdot e_{1}\right) e_{0} .
$$

Defining

$$
G^{-}=e_{1} \widehat{G} e_{1}=-e_{2}\left(G^{*}\right)^{-1} e_{2},
$$

we multiply (5.49) by $e_{1}$ and use (5.92a) to put it in the form

$$
\begin{aligned}
G\left[\frac{1}{2}\left(1-e_{0}\right)+\right. & \left.\frac{1}{2}\left(1+e_{0}\right) \mathbf{x}_{1}^{2}+\mathbf{x}_{1}\right] G^{-} \\
& =\sigma\left[\frac{1}{2}\left(1-e_{0}\right)+\frac{1}{2}\left(1+e_{0}\right)\left[g\left(\mathbf{x}_{1}\right)\right]^{2}+g\left(\mathbf{x}_{1}\right)\right] .
\end{aligned}
$$

This is the representation in $\mathcal{G}_{n+1}$. Here it is understood that $G$ is in $\operatorname{Spin}(p+1, q+1)$, so $g$ is in $\mathrm{SC}(p, q)$. Equation (5.93b) is the conformal representation obtained by Lounesto and Latvamaa [12]. Evidently it has no advantage over (5.49). It is mentioned here to show how alternative representations are related.

The metric affine group $\mathrm{A}(p, q)$ is a particularly important subgroup of $\mathrm{C}(p, q)$. It is the group of orthogonal transformations and translations of $\mathcal{V}_{n}=\mathcal{V}(p, q)$, so it is represented by versors in the canonical form

$$
S=T_{\mathbf{a}} R
$$

It is represented in the orthogonal group of $\mathcal{V}_{n+2}$ as the stability subgroup of the null vector $e_{+}$. That is to say that $S$ belongs to the subgroup of versors satisfying

$$
S e_{+} \hat{S}=e_{+}
$$

Since $e_{+}$commutes with $S$, we can simplify (5.49) for this subgroup by multiplying it by $e_{+}$and using (5.13) and (5.14) to get

$$
S\left(1+e_{0}+\mathbf{x} e_{+}\right) \hat{S}=1+e_{0}+g(\mathbf{x}) e_{+} .
$$


Note that the scale factor $\sigma=1$ is fixed in the subgroup. Since

$$
S e_{0} \hat{S}=e_{0}
$$

we have the further reduction to the simple canonical form

$$
S\left(1+\mathbf{x} e_{+}\right) \hat{S}=1+g(\mathbf{x}) e_{+},
$$

where

$$
\hat{S}=\widehat{R} T_{a}=\left(R^{*}\right)^{-1} T_{a}
$$

is the spin representation of

$$
g(\mathbf{x})=R \mathbf{x} \widehat{R}+\mathbf{a}
$$

the general form for a metric affine transformation.

Note that the entire group representation $(5.96 \mathrm{a}, \mathrm{b}, \mathrm{c})$ can be obtained formally from $\mathcal{G}_{n}$ by extending the algebra to include an element $e_{+}$which commutes with all other elements and satisfies $e_{+}^{2}=0$. Clifford himself extended the quaternions in exactly that way to describe the Euclidean group of rigid rotations and translations in three dimensions [18]. His unfortunate early death prevented him from integrating that idea into his geometric extension of Grassman algebra; there can be little doubt that he would have done so. Here we see that the appropriate integration of Clifford's great ideas entials the identification of $e_{+}$as a vector in a space of higher dimension. This is more appropriate than alternative representations of the affine group in geometric algebra, because of its embedding in the full conformal group.

The affine group representation $(5.96 \mathrm{a}, \mathrm{b}, \mathrm{c})$ has important applications to physics. When the base algebra $\mathcal{G}_{n}$ is the spacetime algebra $\mathcal{G}(1,3)$, it is a representation of the Poincare group. It is also of interest to note that the factorization

$$
\mathcal{G}(1,3)=\mathcal{G}(2,0) \otimes \mathcal{G}(1,1)
$$

implies that the Lorentz group for spacetime is isomorphic to the conformal group for Euclidean 2-space.

\section{References}

1. Hestenes, D. and Ziegler, R.: Projective geometry with Clifford algebra, Acta Appl. Math. 23 (1991), 25-63.

2. Hestenes, D.: Universal geometric algebra, Simon Stevin 62 (1988), 253-274.

3. Hestenes, D. and Sobczyk, G.: Clifford Algebra to Geometric Calculus, D. Reidel, Dordrecht, 1985.

4. Whitehead, A. N.: A Treatise on Universal Algebra with Applications, Cambridge University Press, Cambridge, 1898 (Reprint: Hafner, New York, 1960), p. 249.

5. Clifford, W. K.: Applications of Grassmann's Extensive Algebra, Amer. J. Math. 1 (1878), 350-358.

6. Hestenes, D.: Spacetime Algebra, Gordon and Breach, New York, 1966, 1987.

7. Porteous, 1.: Topological Geometry, 2nd edn., Cambridge University Press, Cambridge, 1981. 
8. Salingaros, N.: On the classification of Clifford algebras and their relations to spinors in $n$ dimensions, J. Math. Phys. 23 (1982), 1.

9. Li, D., Poole, C. P., and Farach, H. A.: A general method of generating and classifying Clifford algebras, J. Math. Phys. 27 (1986), 1173.

10. Dimakis, A.: A new representation for spinors in real Clifford algebras, in J. S. R. Chisholm and A. K. Common (eds.), Clifford Algebras and their Applications in Mathematical Physics, D. Reidel, Dordrecht, 1986.

11. Angles, P.: Construction de revetements du groupe conforme d'un espace vectoriel muni d'une metrique de type $(p, q)$, Ann. Inst. Henri Poincare 33 (1980), 33.

12. Lounesto, P. and Latvamaa, E.: Conformal transformations and Clifford algebras, Proc. Amer. Math. Soc. 79 (1980), 533.

13. Ahlfors, L. V.: Clifford numbers and Möbius transformations in $R_{n}$, in J. S. R. Chisholm and A. K. Common (eds.), Clifford Algebras and their Applications in Mathematical Physics, D. Reidel, Dordrecht, 1986.

14. Maks, J. G.: Modulo $(1,1)$ periodicity of Clifford algebras and generalized (anti-) Möbius transformations, Thesis, Delft University of Technology, The Netherlands.

15. Crumeyrolle, A.: Orthogonal and Symplectic Clifford Algebras, Kluwer Academic Pubs., Dordrecht, 1990, Chap. 12.

16. Kastrup, H. A.: Gauge properties of the Minkowski space, Phys. Rev. 150 (1966), 183.

17. Lang, S.: $\mathrm{SL}_{2}(R)$, Addison-Wesley, Reading, Mass., 1975.

18. Clifford, W. K.: Preliminary sketch of biquaternions, Proc. Lond. Math. Soc. IV (1973), 381-395. 